\title{
Micromanaging alloimmunity
}

\author{
Mandy L. Ford \\ Emory Transplant Center and Department of Surgery, Emory University, Atlanta, Georgia, USA.
}

\begin{abstract}
Increasing evidence indicates that microbes have a large influence on immune function. Previous studies have linked pathogenic microorganisms with decreased allograft tolerance and subsequent rejection. In this issue of the $J C I$, Lei and colleagues demonstrate that commensal organisms also influence the host response to allograft transplantation. Using murine skin and cardiac transplant models, the authors demonstrate that allograft rejection is accelerated in mice with a normal microbiome compared with germ-free animals and antibiotic-treated mice. The increased graft rejection observed in conventional animals was due to enhanced $\mathrm{T}$ cell priming and was mediated through type I IFN. Together, these results suggest that altering a patient's microbial community prior to transplant could improve allograft acceptance.
\end{abstract}

\section{The microbial influence on alloimmunity}

The ability of microbes to modulate alloimmunity has been well appreciated for the better part of two decades. Seminal studies in the early 2000s revealed the impact of acute and chronic viral infections on the establishment of transplantation tolerance $(1,2)$. Later studies demonstrated that concurrent bacterial infection with pathogens, such as Listeria or Staphylococcus aureus, prevents the induction of tolerance and precipitates the loss of the tolerant state following transplantation (3-6). Further, it is known that the immunological memory generated following environmental exposure to pathogens can negatively affect the success of immunosuppressive and tolerance regimens following transplantation (7, 8). Clinically, acute or persistent viral infections can impair efficacy of immunosuppression and result in poorer outcomes in transplant recipients. All of these investigations have focused on the role of pathogenic organisms in the augmentation of alloimmunity and precipitation of allograft rejection, while the impact of nonpathogenic, commensal organisms on the generation and nature of alloimmune responses has remained relatively unknown. Now, in this issue, Lei and colleagues report that the intestinal microbiome potently influences the magnitude of the alloreactive $\mathrm{T}$ cell response and accelerates allograft rejection in murine models of transplantation (9).

While a few clinical studies have demonstrated alterations in microbiota following transplantation of kidney, lung, liver, and small bowel (10-12), it was not clear whether the observed changes were the cause or effect of the associated transplant outcome. Lei et al. addressed this discrepancy by asking an elegantly simple question: does the presence of normal, commensal microbiota at the time of transplantation alter the kinetics of allograft rejection? Working in a model of minor antigen disparity ( $\mathrm{H}-\mathrm{Y})$, Lei and colleagues interrogated the kinetics of skin graft rejection in germ-free (GF) animals relative to specific pathogen-free (SPF) control mice and showed that rejection was markedly delayed in animals lacking commensal microbiota (9). Further, fecal transplant from SPF mice into GF animals restored the accelerated rejection kinetics, demonstrating that microbial colonization is sufficient to enhance the rigor of the alloimmune response. However, it is not simply

Related Article: p. 2736

Conflict of interest: The author has declared that no conflict of interest exists.

Reference information: / Clin Invest. 2016;126(7):2422-2424. doi:10.1172/JCI88621.

any microbe that can mediate this effect. While treatment of recipient animals with antibiotics prior to transplantation delayed allograft rejection, antibiotic treatment did not actually decrease the total bacterial burden within these animals; instead, it reduced the diversity of the microbiome. It is noteworthy that Lei et al. observed an association between a reduction in microbial diversity and improved allograft survival because previous studies have shown that low diversity associates with increased mortality in the setting of hematopoietic stem cell (HSC) transplantation (13). Together, these studies collectively suggest that diversity itself is not sufficient to predict outcome and instead point toward the idea that specific taxa, when present, can provide the necessary signals to augment alloimmunity.

\section{Systemic effects of commensal bacteria}

An important point is that, in addition to restoring intestinal microbiota, oral gavage of fecal contents into GF mice was also sufficient to restore microbial colonization of the skin (9). Because Lei and colleagues were working in a skin transplant model, it was initially unclear whether the ability of microbiome reconstitution to accelerate skin allograft rejection was the result of systemic alteration of immunity or due to a local effect at the site of the graft. Subsequent experiments revealed that microbial colonization also influenced the rejection kinetics of heterotopic heart allografts, organs that are presumed to be sterile. These data suggest the ability of commensal microbiota to augment alloimmunity functions at a systemic level, providing enhanced $\mathrm{T}$ cell priming and improved $\mathrm{T}$ cell responses at sites distant from tissues actually colonized with microbiota.

The ability of an intact microbiome to enhance allograft rejection occurred through enhanced $\mathrm{T}$ cell priming, as adoptive transfer of marked, transgenic $\mathrm{T}$ cells with an $\mathrm{H}-\mathrm{Y}$ antigen-specific $\mathrm{T}$ cell receptor (TCR) into female recipients of male allografts revealed increased proliferation of these cells in recipients that were pre- 
A

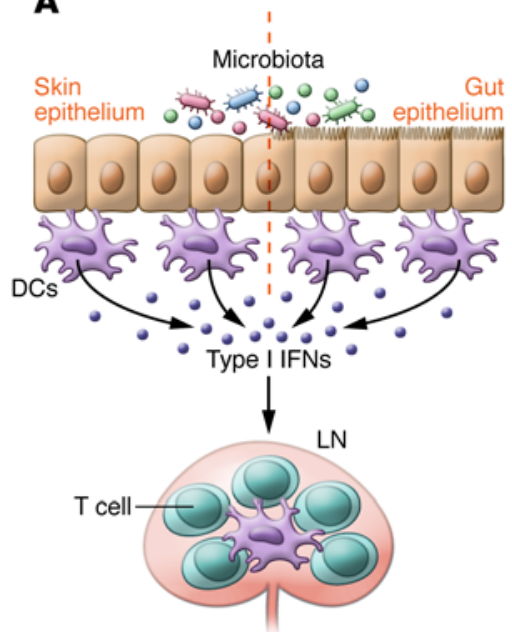

Enhanced T cell priming

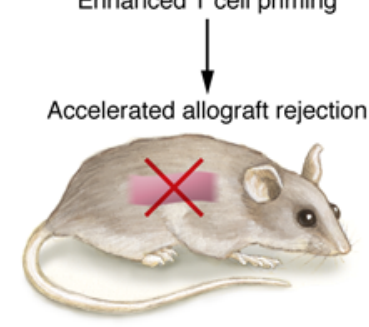

B

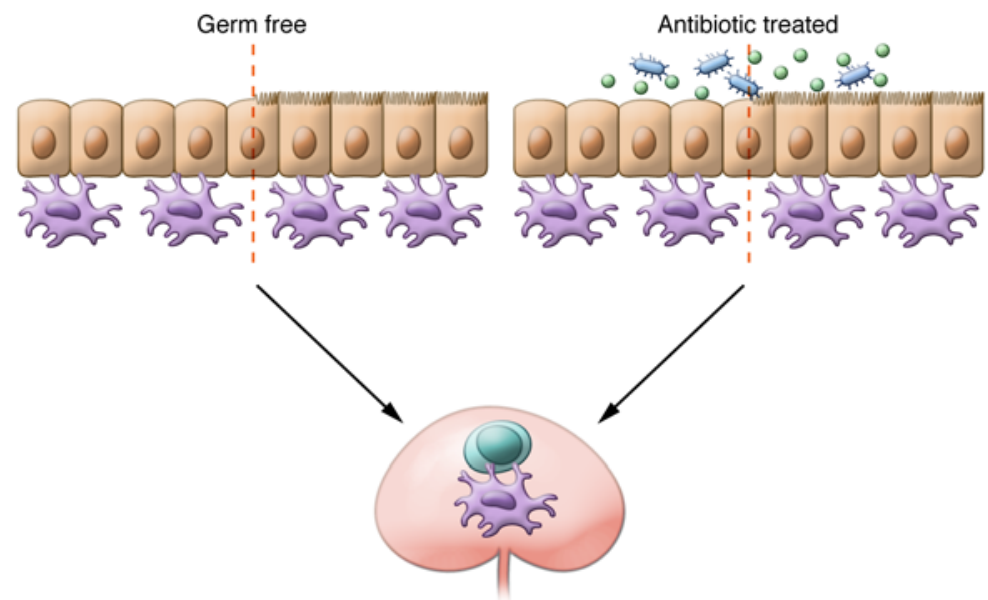

Diminished T cell priming

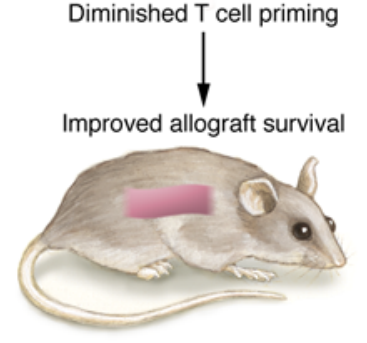

Figure 1. Commensal microbiota affect alloreactive T cell responses by enhancing APC activation in a type I IFN-dependent manner. (A) Exposure of the intestinal and/or skin epithelium to commensal microbes results in systemic alteration of APCs, an effect that can be detected in the LNs. Geneexpression profiles of these APCs reveal upregulation of the type I IFN pathway, and functional studies show that these APCs have increased capacity to prime donor-reactive T cells, which in turn mediate accelerated rejection of an allograft. (B) Allograft survival was improved in GF mice. Alteration of the microbiome with antibiotics also improved allograft survival.

treated with antibiotics (9). Lei et al. further resolved this effect of the microbiome on alloreactive $\mathrm{T}$ cell responses by demonstrating that enhanced $\mathrm{T}$ cell priming is the result of improved antigen-presenting cell (APC) function. Specifically, APCs isolated from skin allografts of SPF-housed animals were better able to stimulate proliferation of transgenic TCR-expressing $\mathrm{T}$ cells in vitro than APCs isolated from skin grafts of antibiotic-treated recipients. It is interesting to note that, despite a profound difference in $\mathrm{T}$ cell-priming capacity between APC populations isolated from control versus antibiotictreated animals, Lei et al. failed to detect any difference in composition of the APC populations (B cells, CD11b ${ }^{+}$monocytes, and CD11 ${ }^{+}$DCs) or in the expression of costimulatory ligands known to be important for the priming of $\mathrm{T}$ cell responses. Instead, the authors found that expression of genes within the type I IFN pathway is reduced in DCs from antibiotic-treated mice. Moreover, survival of skin grafts from type I IFN $\alpha$ Rdeficient males was substantially prolonged in type I IFN $\alpha$ R-deficient females compared with control recipient mice. Importantly, pretreatment of IFN $\alpha$ R-deficient recipients with antibiotics failed to prolong survival as compared with untreated controls. Taken together, these data support a model in which exposure of APCs to commensal microbiota results in enhanced activation of the type I IFN pathway, leading to more robust alloreactive $\mathrm{T}$ cell priming and accelerated allograft rejection (Figure 1).

While the specific APC-priming signals that commensals generate are currently unknown, it should be noted that these effectors can be both initiated and reversed fairly quickly. Impressively, APCs can become poised to activate T cells after only 5 to 7 days of exposure to the microbiota; however, the enhanced stimulatory capacity of microbiome-poised APCs is sufficiently revoked after 10 days of treatment with antibiotics. These findings raise the intriguing notion that because the microbiome is dynamic (14) (with an estimated 30\%-40\% of the population constantly being changed in response to physical activity, lifestyle, bacterial infections, and antibiotic and surgical treatment, ref. 15), the degree of alloreactivity of a given recipient toward a donor graft might also be fluid, fluctuating as the composition of the microbiome changes. Further, it is interesting to speculate that the immune-enhancing signals derived from commensals might be particularly important for alloimmunity, along with autoimmunity and tumor immunity (as opposed to immune responses to viral or bacterial infections), because allogeneic and self tissues themselves do not intrinsically possess DC-licensing TLR ligands the way that pathogen-specific immune responses do (16).

In support of this concept, recent studies by Alegre and colleagues as well as another group have highlighted the importance of the microbiota in shaping immune responsiveness to tumors. Antibiotic treatment or GF conditions resulted in attenuation of tumor-specific $\mathrm{T}$ cell responses relative to those observed in standard SPF animals $(17,18)$. Indeed, specific bacterial species were identified that, when present, were able to augment tumor-specific immu- 
nity, resulting in enhanced tumor clearance. Interestingly, these specific bacteria also influenced the susceptibility of tumors to specific checkpoint inhibitor immunotherapy. One could therefore speculate that the composition of the microbiome may also influence a transplant recipient's relative susceptibility to immunosuppressive therapy following transplantation. However, the situation is even more complex - in the two tumor studies, the presence of $\mathrm{Bac}$ teroides fragilis enhanced susceptibility to anti-CTLA-4 mAbs (18), while the presence of Bifidobacterium enhanced susceptibility to anti-PD-L1 (17). Similarly to the findings of Lei et al., both studies attributed the increased responsiveness to increased DC activation. These findings raise the intriguing possibility that the specific composition of a given individual's microbiome could render that person differentially susceptible to distinct immunotherapeutic interventions. If this principle continues to bear out, one could envision that $16 \mathrm{~S}$ sequencing of a recipient's microbiota prior to transplantation could direct personalized immunosuppression protocols for individual recipients. While specific bacterial species have been identified and implicated in host DC activation and the enhanced priming of antigenspecific immune responses, it is interesting to consider whether commensals always serve to enhance a response or whether there are components of the microbiome that instead function to dampen innate immune activation and mollify subsequent adaptive immune responses.

On a related note, the knowledge that microbiome composition affects the immune system also spurs consideration that posttransplant immunosuppression may influence the commensal microbiota $(19,20)$. A recent report revealed that the human virome (as measured by viral sequences detected in serum) is highly influenced by immunosuppression following transplantation (21). Thus, it is possible that immunosuppression could also have effects on bacterial microbiota, possibly leading to either enhanced or diminished alloimmunity.

\section{Conclusions}

Overall, the study by Lei and colleagues adds to the growing evidence that environmental forces play a paramount role in shaping immunity. For example, a recent study of 210 twins with a range of ages revealed that specific immune parameters showed high heritability during youth; however, the heritability of these traits was markedly lost as subjects aged, suggesting that it is environment and not genetics that primarily dictates immune characteristics of adult humans (22). The notion that nongenetic factors determine immune function is further supported by a recent study that highlighted profound differences in the immune status between laboratory-housed mice and feral or pet-store-reared mice. Specifically, this study demonstrated that feral mice display immune systems more similar to the immune status of adult humans than mice reared in the lab (23). Although not specifically addressed in the study, it is possible that the composition of the microbiomes of feral versus laboratory animals contributed to this effect. If we were to compare the effect of antibiotic treatment on the alloimmune response of feral or pet-store animals, might we find the impact of microbiota on alloimmunity to be even greater than the effect demonstrated by Lei et al.? Perhaps, the answers to questions such as this will illuminate the therapeutic potential of modifying patient microbiota prior to transplantation to limit alloimmunity and promote allograft acceptance.

\section{Acknowledgments}

The author is supported by NIH grants AI104699 and GM113228.

Address correspondence to: MandyL.Ford, 101 Woodruff Circle, Suite 5105, Woodruff Memorial Research Building, Emory University, Atlanta, Georgia 30322, USA. Phone: 404.727.2900; E-mail: mandy.ford @emory.edu.

1. Williams MA, et al. Cutting edge: persistent viral infection prevents tolerance induction and escapes immune control following CD28/ CD40 blockade-based regimen. Jimmunol. 2002;169(10):5387-5391.

2. Williams MA, et al. Characterization of virus-mediated inhibition of mixed chimerism and allospecific tolerance. JImmunol. 2001;167(9):4987-4995.

3. Wang $\mathrm{T}$, et al. Infection with the intracellular bacterium, Listeria monocytogenes, overrides established tolerance in a mouse cardiac allograft model. Am J Transplant. 2010;10(7):1524-1533.

4. Wang T, et al. Prevention of allograft tolerance by bacterial infection with Listeria monocytogenes. JImmunol. 2008;180(9):5991-5999.

5. Ahmed EB, Wang T, Daniels M, Alegre ML,
Chong AS. IL-6 induced by Staphylococcus aureus infection prevents the induction of skin allograft acceptance in mice. Am J Transplant. 2011;11(5):936-946.

6. Miller ML, et al. Spontaneous restoration of transplantation tolerance after acute rejection. Nat Commun. 2015;6:7566.

7. Adams AB, et al. Heterologous immunity provides a potent barrier to transplantation tolerance. J Clin Invest. 2003;111(12):1887-1895.

8. Nadazdin O, et al. Host alloreactive memory T cells influence tolerance to kidney allografts in nonhuman primates. Sci Transl Med. 2011;3(86):86ra51.

9. Lei Y, et al. The composition of the microbiota modulates allograft rejection. J Clin Invest. 2016;126(7):2736-2744.

10. Dickson RP, et al. Changes in the lung microbiome following lung transplantation include the emergence of two distinct Pseudomonas species with distinct clinical associations. PLoS One. 2014;9(5):e97214.

11. Fricke WF, Maddox C, Song Y, Bromberg JS. Human microbiota characterization in the course of renal transplantation. Am J Transplant. 2014;14(2):416-427.

12. Lee JR, et al. Gut microbial community structure and complications after kidney transplantation: a pilot study. Transplantation. 2014;98(7):697-705.

13. Taur Y, et al. The effects of intestinal tract bacterial diversity on mortality following allogeneic hematopoietic stem cell transplantation. Blood. 2014;124(7):1174-1182.

14. Longman RS, Littman DR. The functional impact of the intestinal microbiome on mucosal immunity and systemic autoimmunity. Curr Opin Rheumatol. 2015;27(4):381-387.

15. Kashtanova DA, Popenko AS, Tkacheva ON, Tyakht AB, Alexeev DG, Boytsov SA. Association between the gut microbiota and diet: Fetal life, early childhood, and further life. Nutrition. 2016;32(6):620-627.

16. Ford ML. T cell cosignaling molecules in transplantation. Immunity. 2016;44(5):1020-1033.

17. Sivan A, et al. Commensal Bifidobacterium promotes antitumor immunity and facilitates anti-PDL1 efficacy. Science. 2015;350(6264):1084-1089.

18. Vétizou M, et al. Anticancer immunotherapy by CTLA- 4 blockade relies on the gut microbiota. Science. 2015;350(6264):1079-1084.

19. Fishman JA, Thomson AW. Clinical implications of basic science discoveries: immune homeostasis and the microbiome-dietary and therapeutic modulation and implications for transplantation. Am J Transplant. 2015;15(7):1755-1758.

20. Nellore A, Fishman JA. The microbiome, systemic immune function, and allotransplantation. Clin Microbiol Rev. 2016;29(1):191-199.

21. De Vlaminck I, et al. Temporal response of the human virome to immunosuppression and antiviral therapy. Cell. 2013;155(5):1178-1187.

22. Brodin $P$, et al. Variation in the human immune system is largely driven by non-heritable influences. Cell. 2015;160(1-2):37-47.

23. Beura LK, et al. Normalizing the environment recapitulates adult human immune traits in laboratory mice. Nature. 2016;532(7600):512-516. 